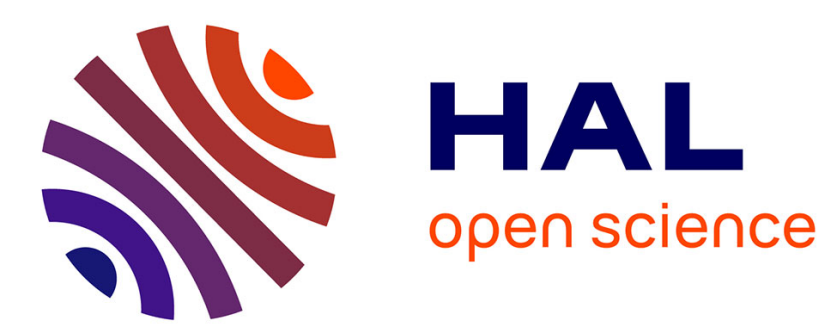

\title{
Du discours juridique à son application: liberté de choix entre une éducation bilingue et une éducation oraliste pour les sourds
}

Hervé Benoit, Murielle Mauguin

\section{- To cite this version:}

Hervé Benoit, Murielle Mauguin. Du discours juridique à son application: liberté de choix entre une éducation bilingue et une éducation oraliste pour les sourds. La nouvelle revue de l'adaptation et de la scolarisation, 2016, 75, pp.31-46. hal-01663049

\section{HAL Id: hal-01663049 \\ https: / hal-inshea.archives-ouvertes.fr/hal-01663049}

Submitted on 13 Dec 2017

HAL is a multi-disciplinary open access archive for the deposit and dissemination of scientific research documents, whether they are published or not. The documents may come from teaching and research institutions in France or abroad, or from public or private research centers.
L'archive ouverte pluridisciplinaire $\mathbf{H A L}$, est destinée au dépôt et à la diffusion de documents scientifiques de niveau recherche, publiés ou non, émanant des établissements d'enseignement et de recherche français ou étrangers, des laboratoires publics ou privés. 


\section{Du discours juridique à son application: liberté de choix entre une éducation bilingue et une éducation oraliste pour les sourds

\author{
Hervé BENOIT \\ Maître de conférences en sciences de l'éducation \\ INS HEA - Université Paris Lumières - Grhapes (EA 7287) \\ Murielle MAUGUIN \\ Maître en conférences en droit public \\ INS HEA - Université Paris Lumières - Grhapes (EA 7287)
}

Résumé: Le processus d'élaboration des politiques publiques relatives aux personnes handicapées comporte différents niveaux. Peuvent ainsi être distingués la construction du problème public, la production de la politique publique, la mise en œuvre et la consommation du produit par les acteurs ${ }^{1}$. Cet article a pour objet d'interroger l'arrière-plan discursif, conceptuel et historique dans lequel s'enracinent, en France, le discours juridique et la politique publique concernant la place et le rôle de la LSF (Langue des signes française) dans l'éducation des jeunes sourds et de montrer en quoi le contexte de controverses qui a marqué historiquement la construction de ce problème public reste présent dans le filigrane des textes juridiques, au point de produire des discordances intertextuelles dont l'effet est tout à la fois de poser un droit pour tous et dans le même temps de restreindre son application. L'ambiguïté juridique qui en résulte constitue le terreau d'un droit souterrain qui semble renvoyer, en l'occurrence, à un interdit idéologique indépassable.

Mots-clés: Discours juridique - Droit souterrain - Éducation bilingue français/LSF - Épistémè - Éducation oraliste - Liberté de choix - Médicalisation - Politique publique - Surdité.

From legal discourse to application: freedom of choice between a bilingual education and an oral education for the deaf children

Summary: The process of developing public policies on disability has different levels. Can thus be distinguished building public problem, production of public policy, implementation and consumption of the product by stakeholders. This paper is intended to examine the discursive, conceptual and historical background in which are rooted in France, legal discourse and public policy, regarding the place and role of the FSL (French Sign Language) in educating young deaf. The aim is to show how the context of controversy that has historically marked the construction of this public problem is present in the watermark of legal texts, to the point of producing intertextual discrepancies the effect of which is to establish a right for all and at the same time restrict its application. The legal ambiguity that results is the breeding ground for an underground law which appears to refer, in this case, an unsurpassable ideological prohibition.

Keywords: Bilingual education French-FSL - Deafness - Épistémè - Freedom of choice - Legal discourse - Oral education - Medicalization - Public policy - Underground law.

1. Prémisses du projet de recherche en cours de l'Enap (École nationale d'administration publique, Québec) et de I'INS HEA sur l'accueil à l'université des étudiants handicapés. 


\section{INTRODUCTION}

Les textes législatifs et réglementaires - que nous désignerons dans cet article sous l'expression discours juridique - relatifs à l'éducation des jeunes sourds se caractérisent historiquement par l'instauration d'un droit pour les parents à la liberté de choix, au titre de l'article 33 de la loi 91-73 ${ }^{1}$ du 18 janvier 1991, "entre une communication bilingue - langue des signes et français - et une communication orale ". Ce nouveau droit correspondait concrètement à une rupture par rapport à la réglementation antérieure (annexe XXIV quater ${ }^{2}$ au décret du 9 mars 1956), dans laquelle la pratique de la LSF (Langue des signes française) n'était mentionnée que comme une "éventualité ${ }^{3}$ " dont le déclenchement relevait de la compétence des responsables éducatifs des établissements médico-sociaux d'accueil. On notera néanmoins que cette liberté a été ensuite fortement encadrée par le décret d'application du 8 mars 1992, qui en limitait l'usage " aux jeunes sourds dont la déficience auditive entraîne des troubles de la communication nécessitant le recours à des techniques spécialisées pour l'apprentissage du français, I'acquisition des connaissances, la formation professionnelle et l'accès à l'autonomie sociale ${ }^{4}$ " (Benoit, 2003).

La loi 2005-102 du 11 février 2005 a confirmé "la liberté de choix entre une communication bilingue, langue des signes et langue française, et une communication en langue française ${ }^{5}$ " et y a ajouté la reconnaissance de la LSF comme langue de la République, de laquelle et dans laquelle " tout élève concerné doit pouvoir recevoir un enseignement ${ }^{6}$ ". Cette seconde disposition entraînait une seconde rupture, puisqu'elle faisait dès lors obligation au système éducatif de mettre en place des programmes et des modalités d'enseignement de la LSF et en LSF, ce qui avait été longtemps considéré par le service public de l'Éducation nationale à la fois comme exorbitant les possibilités d'aménagement de la scolarité et relevant de la prise en charge médico-éducative (Benoit, 2009).

Dans ce contexte marqué par l'empreinte profonde de controverses historiques entre, d'une part, la communauté des sourds et les défenseurs de la LSF et de la culture Sourde ${ }^{7}$ et, d'autre part, les tenants de l'approche médicale de la surdité comme pathologie, pour lesquels le traitement chirurgical par implant cochléaire

1. Loi 91-73 du 18 janvier 1991 portant dispositions relatives à la santé publique et aux assurances sociales: "Dans l'éducation des jeunes sourds, la liberté de choix entre une communication bilingue - langue des signes et français - et une communication orale est de droit. "

2. Décret n 88-423 du 22 avril 1988 remplaçant l'annexe XXIV quater au décret du 9 mars 1956 modifié fixant les conditions techniques d'agrément des établissements privés de cure et de prévention pour les soins aux assurés sociaux par deux annexes concernant, l'une, les conditions techniques d'autorisation des établissements et services prenant en charge des enfants atteints de déficience auditive grave, I'autre, les établissements et services prenant en charge des enfants atteints de déficience visuelle grave ou de cécité, Bulletin officiel du ministère des Affaires sociales et de l'Emploi, fascicule spécial n 88-17 bis, p. 71-77.

3. Ibidem, article 2, p. 73.

4. Décret $n^{\circ}$ 92-1132 du 8 octobre 1992, relatif à l'éducation des jeunes sourds et fixant les conditions d'application de l'article 33 de la loi 91-73 du 18 janvier 1991 portant dispositions relatives à la santé publique et aux assurances sociales.

5. Article L 112-3 du Code de l'éducation.

6. Article L.312-9-1 du Code de l'éducation.

7. Pour citer quelques contemporains: Bernard Mottez, Henri Harry Markowicz, Alfredo Corrado, Emmanuelle Laborit, Philippe Lamothe, Christian Deck, Christian Cuxac, Yves Delaporte, Jean Dagron, Patrice Dalle, André Meynard, Cyril Courtin, Fabrice Bertin, Patrick Masri, Victor Abbou, Guy Bouchauveau, Levent Beskardès, Olivier Schetrit, Marie-Thérèse L'Huillier. 
justifie la préférence de l'éducation oraliste ${ }^{8}$, notre propos est ici d'analyser l'état actuel de la réglementation, c'est-à-dire ce que nous appelons le discours juridique, à propos de l'éducation des jeunes sourds, au regard des "dispositifs discursifs ${ }^{9}$ " (Foucault, 2001, p. 301 ; 2008, p. 13) antagoniques qui constituent les soubassements des pratiques pédagogiques et institutionnelles dans ce domaine. Notre première hypothèse est que les fortes tensions entre les différentes séries de discours éducatifs et idéologiques qui traversent le champ de la surdité, s'inscrivent souterrainement dans le discours juridique, au sein duquel il est possible de repérer leurs traces, comme en filigrane. Notre seconde hypothèse est que se constitue ainsi un discours apocryphe ${ }^{10}$ qui pourrait être à l'origine de l'ambiguïté des politiques publiques qui, au moment même où elles reconnaissent la langue des signes, s'attachent en fait à " faire taire l'enfance sourde " (Meynard, 2010a, p. 21) et à " médicaliser sa parole " (/bid., 2010b). Tout semble se passer comme si des discordances intertextuelles au sein même du discours juridique avaient pour effet tout à la fois de poser un droit pour tous et dans le même temps de restreindre son application. L'amphibologie juridique qui en résulte constitue le terreau d'un droit souterrain qui semble renvoyer, en l'occurrence, à un interdit idéologique indépassable.

\section{CONTEXTE DE L'ÉTUDE}

Le rapport intitulé Les conditions de mise en œuvre des projets linguistiques des jeunes sourds et la qualité de leur parcours, remis en mars 2016 par I'Inspection générale des Affaires sociales et l'Inspection générale de l'Éducation nationale à leurs ministres de tutelle respectifs, apporte un éclairage utile au contexte de la présente étude, non seulement parce qu'il donne des informations sur les modes de prise en charge (dans le secteur médico-social) et de scolarisation (dans le système éducatif) des jeunes sourds, mais encore et surtout parce qu'il apparaît comme le reflet, à travers les commentaires et les recommandations des auteurs (Pierre Naves, Gilles Pétreault et Laurent Brisset), à un moment donné de son évolution, de l'état du positionnement institutionnel de l'Éducation nationale et des Affaires sociales face à cette question très controversée de l'éducation des jeunes sourds. II est d'ailleurs significatif que l'extrême intensité des débats qui, jusqu'à aujourd'hui, ont marqué ce sujet, depuis que le Congrès de Milan en 1880 eut affirmé que la méthode orale devait être préférée à la méthode gestuelle et que le gouvernement français eut appliqué à la lettre cette résolution (Encrevé, 2008, p. 83), ne soit mentionnée qu'en creux et de manière allusive, dans des expressions comme " une compréhension partagée " de "la place légitime " de la LSF (p. 33) ou comme "la place de la LSF est maintenant largement reconnue » (p. 59), qui renvoient au souhait de ne pas

8. Position majoritairement défendue par le corps médical (oto-rhino-laryngologistes, audio-prothésistes et par l'association Acfos (Action connaissance formation pour la surdité).

9. Les dispositifs discursifs, ou épistémès, tels que définis pas Michel Foucault dans Les mots et les choses, sont des formations spécifiquement discursives et peuvent être définies comme le sol épistémologique envisagé sous l'angle des " configurations qui ont donné lieu aux formes diverses de la connaissance empirique " (Foucault, 2008, p. 13).

10. Ce terme n'est pas utilisé par Michel Foucault. Nous l'employons dans sons sens étymologique de caché, tenu secret, qui n'est pas authentique, (Rey, Dictionnaire historique de la langue française). Voir Benoit, 2013, p. 58) 
" reprendre I'histoire d'un débat passionné et complexe sur la place d'une langue des signes dans les apprentissages" (p. 35) présenté plus loin comme "la querelle sur la langue des sourds » (p. 36).

De son côté, la HAS (Haute autorité de santé), qui a publié en décembre 2009 une recommandation de bonnes pratiques destinée à éclairer le choix des parents vers une communication en LSF ou vers I'oralisation avec ou sans implant cochléaire, indique qu'en "l'état actuel des connaissances scientifiques, il n'a pas été possible de recueillir un consensus entre les différents acteurs pour proposer de recommander une approche plutôt qu'une autre lapproche audiophonatoire ou approche visuogestuelle) » (2009, p. 10). À cet égard, le rapport des Inspecteurs généraux remarque que la position de la HAS " n'a été l'objet d'aucune critique » et que les réponses des responsables rencontrés "ont confirmé qu'il s'agissait d'un document opérationnel et équilibré, qui faisait consensus et constituait une référence toujours valide ". Dans cette situation paradoxale où c'est l'absence de consensus qui fait consensus, l'enjeu des politiques éducatives et sanitaires semble relever d'un équilibre à tenir entre deux options dont la validité est également reconnue par l'autorité de santé.

Au-delà de la période de 0 à 6 ans, sur laquelle portent les recommandations de la HAS, le rapport de I'Igen et de l'Igas, après avoir rappelé que la loi du 11 février 2005 reconnaît la Langue des signes française (LSF) comme une langue à part entière et qu'elle établit le droit des familles "à choisir entre une éducation avec une communication bilingue (LSF et langue française) ou en langue française " (p. 3), affirme néanmoins que "l'enjeu consiste sans doute à structurer suffisamment I'acquisition de la LSF tout au long du parcours de l'enfant sourd pour lui permettre de s'exprimer le plus efficacement possible dans les deux modes (français oral et LSF) » (p. 20-21). Ainsi les rapporteurs s'écartent-ils sensiblement de la nomenclature applicable au Projet personnalisé de scolarisation (PPS) mentionnée en annexe 2 de l'arrêté"11 du 6 février 2015 qui distingue trois "modes de communication ": 1.1. Langue française; 1.2. Langue française avec LPC (Langue française parlée complétée) ; 1.3. Communication bilingue (Langue française + LSF : Langue des signes française), dans laquelle le " français oral " n'est pas mentionné. Le fait de présenter l'acquisition de la LSF comme une propédeutique à l'oralisation introduit, à côté du bilinguisme une notion distincte, issue de la culture professionnelle des acteurs du secteur médico-social traditionnellement favorable à l'éducation oraliste, celle de la bimodalité, qui ne figure dans aucune disposition législative. Au bilinguisme français + LSF, se substitue insensiblement au fil de la rédaction une nouvelle catégorie de nomenclature, qualifiée de bilinguisme bimodal (p. 28) et adossée à la bimodalité bilingue (p. 36), qui transparaît dans le rapport à travers les expressions " offres linguistiques bilingues et bimodales " et " mode bilingue / bimodal " (p. 27), " choix du bilingue - bimodal » (p. 28), " pratiques bilingues et bimodales " (p. 38), " approche bimodale» (p. 60). La recommandation 5 (p. 60) qui fait suite à l'idée que

11. Arrêté du 6 février 2015 relatif au document formalisant le Projet personnalisé de scolarisation (PPS), BOEN n 8 du 19 février 2015 (http://cache.media.education.gouv.fr//file/8/70/0/BO_08_MEN-19-22015_393700.pdf). 
"les pratiques d'enseignement bilingues et bimodales sont à encourager " (p. 36) conclut à la nécessité de la mise en place d'un " schéma national de formations bilingues favorisant une approche bimodale ". Dans ces conditions, il est clair que la liberté de choix des parents entre les deux types de "projets linguistiques " se trouve altérée si, dans sa mise en œuvre, l'une des options proposée, celle de la communication bilingue, est dénaturée par confusion avec celle l'éducation oraliste. Or ce glissement progressif de la notion juridique de bilinguisme français LSF et de communication bilingue (LSF et français écrit ${ }^{12}$ ) à celle, jugée plus pragmatique, de bilinguisme bimodal (encore que les deux termes de cette expression soient souvent rapprochés, mais jamais formellement juxtaposés dans le rapport) est susceptible de générer du "droit souterrain " (Warsmann, 2008, p. 112; Conseil d'État, 2006, p. 276), dans la mesure où les avis des inspections générales ont vocation à inspirer les rédacteurs des futures circulaires et notes de service, mais aussi de faire autorité auprès des praticiens de terrain.

Le premier problème que pose une telle orientation de la politique éducative est tout d'abord de se trouver en contradiction assez nette avec les programmes de $\mathrm{LSF}^{13}$ publiés en 2008 qui précisent que "la pratique de la langue des signes française tient lieu d'équivalent de communication orale, et la langue française écrite tient lieu de langue écrite " et que s'il est souhaité " autant que faire se peut, que les jeunes sourds aient un accès même limité à l'oral ", ils " ne seront pas évalués sur leurs compétences en français oral qui, par conséquent, ne sera pas systématiquement enseigné » (p. 12). Le second est qu'elle pourrait contribuer à affaiblir la dimension bilingue des Pôles d'accompagnements à la scolarisation des élèves sourds ${ }^{14}$ (Pass), déjà fragile de l'aveu même des inspecteurs généraux (p. 30 et 31), puisque les enseignements de LSF qui y sont dispensés par des professeurs vacataires ou titulaires du Capes de $L S F^{15}$ concernent paradoxalement des lycéens entendants préparant l'option LSF du baccalauréat (p. 12-13), alors même que l'on aurait pu penser que leur public naturel serait les élèves (sourds) ayant suivi le cursus d'apprentissage bilingue depuis l'école primaire et le collège et dont la LSF est la langue première.

Le rapport semble ainsi s'inscrire dans le renversement de la logique des pôles LSF, initialement mis en place en application de l'arrêté de juillet 2008, pour favoriser un enseignement de la LSF et en LSF tout au long du parcours scolaire de la maternelle à l'université, auxquels sont substitués, en 2010, les Pass, destinés à promouvoir " un renforcement de l'enseignement du français » (p. 14); il accentue encore cette tendance puisque la circulaire de 2010 indiquait que ce renforcement devait concerner " l'écrit pour les élèves ayant fait le choix d'une communication bilingue, (l')écrit et (I')oral pour les élèves ayant fait le choix d'une communication en langue française ".

12. Ainsi définie dans la circulaire n²010-068 du 28 mai 2010 portant Organisation des "Pôles pour l'accompagnement à la scolarisation des jeunes sourds".

13. Arrêté du 15 juillet 2008 portant Enseignement de la Langue des signes française à l'école primaire.

14. Cf. note 4

15. Le Capes de LSF a été créé en 2009. 


\section{CADRE THÉORIQUE}

\section{La théorie des " épistémès "}

L'analyse de l'arrière-plan discursif et conceptuel dans lequel s'enracinent, en France, la construction du problème public et la production de la politique publique de la liberté de choix entre l'oralisme et le bilinguisme français LSF dans l'éducation des sourds s'inscrit dans une perspective poststructuraliste. On s'intéresse aux soubassements discursifs des controverses qui ont marqué historiquement la construction de ce problème public. Ces configurations discursives sous-jacentes, nommées "épistémès " par Michel Foucault (2008, p. 13), constituent des réseaux anonymes sous-jacents à l'histoire des discours et s'organisent en territoires d'énoncés antagoniques dont il est possible de cerner à la fois les relations et les principes de fonctionnement. C'est dans ce cadre que nous faisons l'hypothèse que le discours juridique n'est pas indépendant de ces formations discursives et que l'on retrouve en filigrane de la réglementation actuelle sur la liberté de choix des parents entre les deux types d"éducation des jeunes sourds des traces de ces antagonismes discursifs sous la forme de discordances juridiques intertextuelles.

Dans trois précédentes études (Benoit, 2014a; Benoit 2014b, Benoit, 2014c), nous avons identifié, d'un point de vue généalogique, quatre séries de discours dans le champ du handicap: d'une part les discours de pathologisation des difficultés sociales et les discours médicaux de remédiation du trouble, et, d'autre part les discours de l'inclusion sociale et scolaire, qui, sous l'effet de discours catalyseurs, les discours institutionnels injonctifs de collaboration, vont percuter les deux séries de discours précédentes et provoquer une hybridation des concepts de besoins éducatifs particuliers et d'inclusion scolaire qui, le premier, croisant celui de trouble, se trouve vidé de son sens situationnel, et, le second, croisant celui d'intégration, se trouve soumis à la condition de capacité d'adaptation et découpé en tranches temporelles dénommées couramment temps d'inclusion.

Dans le domaine particulier de la surdité et de l'éducation des jeunes sourds, le processus de collision des flux de discours s'actualise dans la rencontre des discours médicaux sur le déficit auditif et des discours socio-anthropologiques sur la langue et la culture Sourdes qui constituent la signature des Deaf studies. Pour Andrea Benvenuto et Alexis Karacostas (2012), deux grands discours encadrent et structurent aujourd'hui les savoirs et les pratiques relatifs à l'éducation des jeunes sourds:

" le discours de la déficience, de nature médico-pédagogique, centré sur l'oreille

défaillante et l'enseignement de la parole orale et le discours socio-anthropologique de la différence, centré sur la langue des signes et la culture sourde".

À la confluence de ces deux formations discursives, le concept de S(s)ourd connaît lui aussi une scission qui se manifeste à travers l'ambivalence majuscule vs minuscule de la lettre initiale.

\section{"Les forces créatrices du droit "}

Du point de vue juridique cette grille d'analyse discursive renvoie aux travaux du juriste Georges Ripert, dans son ouvrage Les forces créatrices du droit. Ainsi Paul Roubier (1955, p. 878), commentant l'approche de Ripert, évoque-t-il « toutes les 
forces qui travaillent à l'élaboration du droit positif " parmi lesquelles sont en conflit, selon Ripert, des "forces économiques ou religieuses ou politiques qui travaillent, soit dans un sens conservateur, soit dans un sens réformateur ".

La trace de cette lutte entre des éléments discursifs et idéologiques opposés dans le discours juridique fait précisément l'objet de cette étude centrée sur la liberté de choix des parents entre les deux types d'éducation oraliste ou bilingue des jeunes sourds dans les textes législatifs et réglementaires. Pour reprendre les propos de Paul Roubier, elle s'inscrit bien pour nous dans la lignée du projet de Georges Ripert, de montrer "par le jeu de quelles forces les règles juridiques ont été créées " et de ne pas nous arrêter " au seuil de la naissance des lois", mais d'aller plus loin et d'étudier "la mise en œuvre des lois, par le gouvernement, ou par l'autorité judiciaire, ou encore par les intéressés eux-mêmes " (Ibid.).

\section{La figure du " déni "}

De la confrontation des approches socio-anthropologiques de la surdité et des dispositifs de normalisation, adossés aux discours de pathologisation de la communication gestuelle, naît un principe générateur d'hybridation des discours qui est la figure du déni. André Meynard (2010a, p. 22) évoque, à propos du risque de mise à l'écart des langues signées, cette notion freudienne de Verleugnung, processus psychique par lequel on prend connaissance et l'on admet l'existence d'une réalité, tout en conservant intacte la croyance inverse qui l'invalide. Ainsi peut-on connaitre sans reconnaître, c'est-à-dire refuser de prendre connaissance et particulièrement de tirer les conséquences au niveau symbolique (Bon, 2004) d'une réalité que l'on perçoit ou d'un acte que l'on commet. On est proche de la notion juridique de non-lieu, qui peut correspondre à un déni de justice.

Dans le domaine de la fiction cinématographique, l'expression utilisée par Octave Mannoni (1969) " Je sais bien, mais quand même... " dans le titre d'un article où il analyse le spectateur comme un sujet clivé vis-à-vis du spectacle auquel il assiste, permet de se représenter le fonctionnement de la figure du déni comme générateur et organisateur de discours et de dispositif de type social. De même que le spectateur est clivé en ce sens qu'il n'y croit pas mais quand même - et cela sans qu'il y ait antagonisme entre celui qui, en lui, y croit, et celui qui n'y croit pas, le discours et le dispositif articulent en eux-mêmes des éléments ou des objets contradictoires, en s'abstenant de prendre en charge ces contradictions, de sorte que tout se passe comme si elles n'existaient pas.

\section{LES DISCOURS MÉDICO-SOCIAUX FACE AUX DISCOURS SOCIO-ANTHROPOLOGIQUES SUR LA SURDITÉ 16}

\section{Formation des discours médico-éducatifs}

La rencontre au cours du XIXe siècle de la médecine et de la pédagogie sur le terrain de la surdité est consécutive à l'entrée des médecins dans les institutions de sourds-

16. Les éléments de cette partie, indispensables à la compréhension de la démarche de confrontation des "épistémès " et du discours juridique, sont repris de l'article publié en 2014 (Benoit, 2014a), qui peut être consulté pour de plus amples développements de la notion d'hybridation. 
muets - dont la nomination en 1800 par l'abbé Sicard de Jean-Marc Gaspard Itard comme premier médecin de l'Institut national des jeunes sourds (INJS) de Paris est emblématique. J.-M. Itard s'efforça dans le cadre de l'éducation physiologique, de s'appuyer sur les restes auditifs des élèves pour les démutiser en leur enseignant I'articulation artificielle. À ce titre, il se trouvait en ce début de XIX à contre-courant des orientations pédagogiques de son temps, favorables au développement du langage mimique, c'est-à-dire de la communication gestuelle. La prééminence du discours médico-pédagogique sur la surdité se trouve historiquement et institutionnellement installée à la suite du Congrès de Milan qui se déroule du 6 au 11 septembre 1880. Les participants à ce congrès s'étant accordés sur la préférence de "la méthode orale pure " (Compte rendu, 1881) décident de mettre un terme définitif, parce qu'ils la considèrent comme nuisible, à l'éducation des sourds en langue des signes, qui avait dominé la première moitié du siècle (Benoit, 2014a).

Le nouveau programme d'enseignement du 13 juillet 1889, qui officialise la méthode orale pure comme seule méthode d'enseignement pour les élèves sourds (Séguillon, 1994, p. 14), est l'une des étapes du long processus de médicalisation de l'éducation des sourds, engagé par Jean Itard et poursuivi au long du XIXe siècle par ses successeurs, les docteurs Ménière, Ladreit de la Charrière et Leroux. Par vagues successives de réorganisation (1827, 1859), des dispositions pratiques vont progressivement marginaliser les professeurs sourds et accorder une place croissante à la parole dans la scolarité des jeunes sourds. La médecine, en optant pour une vision déficitaire de la communication gestuelle, apporte ainsi son concours au " dispositif stratégique 17 " destiné à bannir totalement et définitivement la langue des signes de l'éducation des jeunes sourds, dans le cadre de la coercition normalisatrice de la société, et prépare, dès cette époque, le processus de substitution de la norme à la loi qu'évoque Michel Foucault en 1976:

"Aujourd'hui, la médecine est dotée d'un pouvoir autoritaire aux fonctions normalisatrices qui vont bien au-delà de l'existence des maladies et de la demande du malade [...] On peut affirmer que les médecins du XXe siècle sont en train d'inventer une société de la norme et non de la loi. " (Foucault, 2001b, p. 50).

\section{Formation des discours socio-anthropologiques}

Ce sont des travaux de linguistes, ceux de William C. Stokoe professeur à I'université Gallaudet de 1955 à 1970, puis de Christian Cuxac en France (2000), de sociologues comme Bernard Mottez dans les années 1970, puis plus tard de philosophes et d'anthopologues comme H.-J. Stiker $(1999,2003)$ et Charles Gardou (2002, 2005), de psychanalystes comme André Meynard (1995, 2008, 2010), d'artistes comme Emmanuelle Laborit, qui vont sortir progressivement les langues signées du ghetto d'invisibilité où elles se trouvaient reléguées et leur permettre de refaire surface dans I'espace public.

17. Un " dispositif stratégique " pour Michel Foucault, (2001a, p. 299) combine et articule les formations discursives avec d'autres éléments, dits et non dits, et constituer une structure d'éléments hétérogènes caractérisés par la " prévalence d'un objectif stratégique ", dont la fonction est de répondre à un moment donné à une urgence de type social. 
Après cent ans de quasi-interdiction de la langue des signes, les années 1970 apportent un renouveau du regard porté sur les sourds, grâce notamment à Bernard Mottez, Harry Markowicz, à la publication de la revue Coup d'œil, à la création d'IVT (International Visual Theater) en 1976 par Alfredo Corrado et Jean Grémion. Dans ce bouillonnement culturel, le voyage organisé en 1978 par Bernard Mottez à I'université Gallaudet de Washington, va permettre à de nombreux sourds de découvrir qu'aux États-Unis I'usage de la langue des signes dans l'enseignement supérieur est reconnu et ne fait l'objet d'aucune stigmatisation.

Le cycle de l'opprobre et de l'invisibilité, dans lequel se trouvaient enfermés les sourds, parce qu'ils se percevaient eux-mêmes de manière négative et n'osaient pas signer devant des entendants, est rompu (Laborit, 2010, p. 62). Les sourds deviennent des sujets linguistiques, la LSF accède au statut de langue à part entière, qui sera reconnu par la loi du 11 février 2005, et non plus de code de suppléance, et la conscience de constituer une communauté culturelle se fait jour (Benoit, 2014a). Tout au long des 30 années qui séparent les années 1970 du milieu des années 2000, les discours de type socio-anthropologique se construisent en s'articulant à la notion centrale de pertinence socio-linguistique - et non pas physiologique - de la surdité au regard du développement de la langue des signes. Le besoin de l'enfant sourd de communiquer avec son entourage donne lieu à une intention sémiotique qui se manifeste à travers un processus d'iconicisation consistant à donner à voir I'information à transmettre grâce à des signes gestuels:

"La forte ressemblance des formes gestuelles montre qu'un processus d'iconicisation de l'expérience a été mis en œuvre et que ce processus se fonde sur la description de contours de formes et/ou la reprise gestuelle iconique de formes saillantes des référents catégorisés. " (Cuxac, 2000, p. 22).

\section{Des discours hybrides fondés sur la figure du " déni "}

À la confluence des deux types de discours, médico-éducatifs et socio-anthropologiques, s'est développée une palette de discours institutionnels hybrides, empruntant à la fois au médical, au pédagogique et au sociolinguistique et marqués par la figure du déni. Cette notion issue de la théorie psychanalytique est ici convoquée, non pas pour expliquer les fonctionnements discursifs, mais comme analyseur des contradictions intra ou intertextuelles. Elle constitue un modèle d'analyse permettant de rendre compte de textes ou de séries de textes qui admettent en leur sein la coexistence d'éléments contradictoires, tout en les passant sous silence.

Ces discours hybrides relatifs à la surdité comportent généralement un paradoxe lié à la nature et au niveau de la reconnaissance de la langue des signes: ce qui est reconnu au niveau politique est le statut linguistique de la LSF, à la fois en tant que discipline scolaire et qu'outil véhiculaire de communication bien adapté aux sourds, en vue de l'accessibilité aux services et aux savoirs. Mais il en va différemment de la reconnaissance sociologique et anthropologique:

"La LSF, non pas réduite au statut de langue adaptée à une population "déficiente,"

mais comprise comme langue vitale d'une communauté singulière et dont les référents sont les sourds. " (Dalle-Nazébi, Garcia, Kerbourc'h, 2009, p. 7-8). 
L'ambiguïté profonde soulignée par ces auteurs se trouve inscrite dans le processus même de reconnaissance de la Langue des signes française, puisque c'est dans une loi portant sur "les personnes handicapées ", celle du 11 février 2005, que son statut de langue à part entière se trouve affirmé.

\section{Un exemple d'hybridation discursive concomitante}

On pourrait par exemple illustrer ce processus en pointant la concomitance, dans un même temps social, d'une loi de 2005 reconnaissant la langue des signes française comme une langue à part entière, assortie d'un droit à l'enseignement pour les "élèves concernés ", et de l'accord donné en 2007 par la Haute autorité de la santé (HAS, 2007, p. 32) à la généralisation d'un " dépistage néonatal de masse " dans les deux jours après la naissance, en vue de pouvoir recourir aux implants cochléaires avant un an pour traiter la maladie-surdité. II est clair qu'un tel dépistage médical s'inscrit en opposition à la perspective d'une éducation fondée sur la communication signée. Ce cas précis ne relève pas toutefois à proprement parler du droit souterrain, puisque le dépistage (d'ordre médical) ne vient pas remettre en cause l'existence de la liberté de choix.

\section{Un exemple d'hybridation discursive simultanée}

Mais on pourra trouver de nombreux exemples où le je sais bien et le quand même ne sont pas disjoints, mais intégrés dans un seul et même discours. Le 13 février 2002, Jack Lang, ministre de l'Éducation nationale prononce un discours (Benoit, 2009 , p. 177) destiné à affirmer, en s'appuyant sur le $C E C R L^{18}$, la légitimité éducative, pédagogique et culturelle de la langue des signes. S'y côtoient les déclarations suivantes:

"1) À la fin du XIXe siècle [...] tous les enfants sourds devaient donc apprendre à parler, que leurs parents l'aient ou non choisi. Ils devenaient ainsi, très souvent, victimes des excès, voire de la violence de leurs éducateurs.

2) [...] je souhaite également saluer et rendre hommage aux parents et aux associations qui font prioritairement le choix de l'acquisition du français oral et écrit. Ils ont administré la preuve qu'une éducation bien adaptée, assortie de toutes les aides thérapeutiques nécessaires, permettait à de jeunes enfants sourds d'acquérir une langue orale de qualité. "

Et cette formule péremptoire et définitive:

«3) Soyons clair: toute définition du bilinguisme qui éliminerait la référence théorique au français oral nous replongerait immédiatement dans les impasses anciennes. » (http://cis.gouv.fr/spip.php?article1898).

\section{Un exemple d'hybridation discursive successive}

Tous les discours du déni, dans ce domaine, ne présentent pas des formes d'hybridation simultanée ou concomitante, certains d'entre eux inscrivent le je sais bien et le quand même dans une chronologie du passage à l'acte et du repentir,

18. Cadre européen commun de référence pour les langues. 
caractéristique d'une hybridation successive. Après un premier texte ${ }^{19}$, publié au BOEN du 4 septembre 2008, instituant des "pôles-ressources en LSF ", constitués d'un " ensemble articulé d'établissements scolaires du premier et du second degré " dans lesquels les "élèves concernés " pourront recevoir tout au long de leur scolarité un enseignement "de et en LSF », un second texte ${ }^{20}$, deux ans plus tard, remplace ce premier dispositif par des "Pôles pour l'accompagnement à la scolarisation des jeunes sourds".

Dans la perspective de notre analyse, ces deux textes ne doivent pas être considérés comme deux discours distincts et opposés, mais comme un seul et même discours hybridé de manière successive, dont la première partie a pour fonction d'instaurer les conditions de possibilité de la seconde.

Ainsi la première circulaire, rappelant que la loi "reconnaît à la langue des signes française (LSF) un statut de langue de la République au même titre que le français", attribue-t-elle en même temps à ce " mode de communication » le rôle de préalable et de vecteur de l'ensemble des apprentissages:

"À partir de l'apprentissage ou de la consolidation de sa connaissance de la

Langue des signes française, l'institution scolaire s'efforce de construire pour chaque élève sourd dont la famille a fait ce choix, un accès graduel au français en s'appuyant d'abord sur le français écrit [...]"

Le destinataire principal de cet enseignement est d'ailleurs clairement identifié en tant qu'élève sourd et non pas en tant que locuteur de LSF, même si le texte élargit le public auquel cet enseignement s'adresse en principe à " tout élève concerné21", (fratries, camarades de classe, environnement proche), ce qui tend à conjuguer la détermination déficitaire - ou compensatoire -, manifestement première, et la pertinence sociale de la LSF, maintenue au second plan, en dépit des apparences novatrices.

Dans ces conditions, il n'est pas surprenant que la circulaire de 2010 remette sur le devant de la scène la question de la scolarisation des jeunes sourds, en substituant dans son objet la question déficitaire à la question linguistique. C'est le principe de liberté de choix éducatif22 des parents au regard de la surdité qui est invoqué pour justifier le renversement de perspective: tout se passe comme si la possibilité pour les parents de préférer l'approche éducative fondée sur la compensation du déficit surdité invalidait de droit l'existence d'un dispositif qui tiendrait sa cohérence d'une approche socio-anthropologique de la langue des signes française comme langue vitale d'une communauté singulière :

"Ces deux modes de communication doivent être possibles dans les établissements scolaires où sont regroupés des élèves sourds [...] La mise en place des premiers pôles [...] a montré le besoin de modifier le concept de ce dispositif et de l'étendre aux élèves sourds dont les parents ont fait

19. Circulaire n² 2008-109 du 21 août 2008, BOEN n 33 du 4 septembre 2008: "Conditions de mise en œuvre du programme de Langue des signes française à l'école primaire ".

20. Circulaire n²010-068 du 28 mai 2010, BOEN n² 25 du 24 juin 2010: "Organisation des "Pôles pour I'accompagnement à la scolarisation des jeunes sourds" ".

21. Article L 312-9-1 du Code de l'Éducation.

22. Article $L$ 112-3 du Code de l'Éducation. 
le choix d'une communication en langue française (écrit et oral), afin de permettre aux familles d'exercer leur libre choix $x^{23}$ entre ces deux modes de communication" (BOEN, 2010).

\section{DISCORDANCES INTERTEXTUELLES DANS LE DISCOURS JURIDIQUE}

On peut retrouver ce processus d'hybridation des discours sur la surdité sous la forme de discordances intertextuelles dans la série de textes réglementaires concernant la liberté de choix des parents entre une communication bilingue français LSF et une communication en langue française dans l'éducation des jeunes sourds.

Les textes sur lesquels porte notre analyse sont, d'une part, les deux articles L.112-3 et L.312-9-1 du Code de l'Éducation qui établissent respectivement le droit pour les jeunes sourds et leurs familles à " la liberté de choix entre une communication bilingue [...], et une communication en langue française " et à "recevoir un enseignement de la LSF ", et, d'autre part, le décret d'application 2006-509 du 3 mai 2006 et I'arrêté du 15 juillet 2008.

Deux principes fondamentaux et complémentaires sont affirmés par la loi 2005-102 sur l'égalité des droits et des chances, la participation et la citoyenneté des personnes handicapés et inscrits dans le Code de l'Éducation:

Article L 112-3: "Dans l'éducation et le parcours scolaire des jeunes sourds, la liberté de choix entre une communication bilingue, langue des signes et langue française, et une communication en langue française est de droit. Un décret en Conseil d'État fixe, d'une part, les conditions d'exercice de ce choix pour les jeunes sourds et leurs familles, d'autre part, les dispositions à prendre par les établissements et services où est assurée l'éducation des jeunes sourds pour garantir l'application de ce choix. "

Article L.312-9-1: "La langue des signes française est reconnue comme une langue à part entière. Tout élève concerné doit pouvoir recevoir un enseignement de la LSF. Le Conseil supérieur de l'éducation veille à favoriser son enseignement. Il est régulièrement informé des conditions de son évaluation. Elle peut être choisie comme épreuve optionnelle aux examens et concours, compris ceux de la formation professionnelle. Sa diffusion dans l'administration est facilitée. " Ces deux principes donneront lieu à deux textes d'application: citons tout d'abord le décret, en date du 15 juillet $2008^{24}$, qui, en instaurant des programmes d'enseignement de la LSF à l'école primaire, se trouve en parfaite cohérence avec l'esprit de la loi puisqu'il garantit au sein de l'École de la République, I'application du choix de l'éducation bilingue français-LSF, et cela en rupture totale par rapport aux pratiques antérieures. Ce décret comporte en annexe les programmes d'enseignement de la LSF à l'école primaire, dispensés aux élèves concernés dans le cadre horaire de l'enseignement du français. Non seulement la LSF n'est plus une simple béquille pédagogique permettant d'apprendre le français oral et écrit dans un cadre d'éducation spéciale, non seulement elle accède au statut de véritable discipline d'enseignement scolaire, mais encore entre-t-elle dans le champ des compétences de " maitrise de

23. Ce sont les auteurs qui soulignent à deux reprises.

24. Arrêté du 15 juillet 2008, BOEN n³3 du 4 septembre 2008. 
la langue ", puisqu'elle est dispensée, pour les jeunes sourds dont c'est la langue naturelle, pendant les heures de français.

En revanche, il en va différemment du décret 2006-509 du 3 mai 2006, qui en reprenant presque sans changement le décret d'application de la loi de 1991 sur la liberté de choix éducatif des jeunes sourds semble au contraire s'inscrire en contradiction avec le principe de liberté de choix, puisqu'il dispose que "le mode de communication adopté par le jeune sourd est inscrit dans le projet de vie [...] après un diagnostic constatant les difficultés d'accès à la communication orale et la nécessité du recours à des modalités adaptées de communication ". On notera d'ailleurs que l'article L 112-3 contient potentiellement sa propre discordance simultanée ou intratextuelle en annonçant que l'exercice de la liberté créée sera encadré juridiquement: " un décret en Conseil d'État fixe les conditions d'exercice de ce choix ".

Ainsi le droit de toute famille d'enfant sourd de librement opter pour une éducation fondée sur une communication bilingue, langue des signes et langue française, se trouve-t-il de fait subordonné à la pose d'un diagnostic médical, non pas de la surdité, mais de son impact sur les possibilités d'accès à la communication orale. La langue des signes, reconnue par la loi comme " une langue à part entière » (L.312-9-1), se trouve ainsi ravalée au rang des "modalités adaptées de communication ». La norme médicale s'immisce donc dans l'application du droit à la liberté de choix éducatif, en la conditionnant à un diagnostic, ce qui correspond au pronostic de Michel Foucault d'une société où la norme prend le pas sur la loi. Dans ces conditions, I'ambiguïté de la réglementation peut entraîner la confiscation par les experts, dans un second temps, du droit initialement attribué par la loi aux familles: la demande d'inscription du bilinguisme français LSF dans le projet de vie d'un enfant sourd pourrait être en effet rejetée par la MDPH (Maison départementale des personnes handicapées), dès lors que le diagnostic de difficulté d'accès à la communication orale n'aurait pas été posé et les chances de succès d'un recours par la famille devant un tribunal administratif seraient quasi nulles, au regard du décret 2006-509.

Le discours juridique se trouve ainsi concerné par un même type d'hybridation successive que celui que nous avions identifié dans le retour à la logique de la déficience lié à la transformation des pôles LSF en Pass et qui marquait l'abandon de la perspective sociolinguistique et culturelle de la langue des signes au profit d'une approche par le handicap et I'oreille défaillante. Cette hybridation prend la forme d'un droit souterrain, dans un contexte dont nous avons vu, à travers l'analyse du rapport des Inspections générales de l'Éducation nationale et des Affaires sociales, qu'il semble particulièrement perméable aux influences du discours médico-éducatif dominant, qui prend appui sur une praxéologie médico-éducative pour suggérer que les deux options se rejoignent et se confondent dans un bilinguisme bimodal ou une bimodalité linguistique, ce qui revient à frapper de nullité et à disqualifier le principe même de l'alternative. À quoi bon en définitive donner le choix aux parents, si les professionnels qui en assurent par la suite la mise en œuvre estiment non fondée la distinction de modalités éducatives sur laquelle il repose? 


\section{POUR CONCLURE}

La mise au jour des discordances intertextuelles dans la série des textes réglementaires concernant la liberté de choix entre une communication bilingue français LSF et une communication en langue française tend à attester la pertinence d'une analyse des interrelations entre les formations discursives qui constituent les soubassements de la construction du problème public, de la production de la politique publique, de la mise en œuvre et de la consommation du produit par les acteurs.

Ces déterminants discursifs, impensés par ceux mêmes qui les déterminent et qu'ils déterminent, semblent dans le domaine de la surdité assigner le discours juridique à un clivage indépassable. On pourrait y voir une forme de sidération du législateur confronté à l'impensable anthropologique au regard d'une norme sociale, et malgré tout contraint de produire un discours injonctif à son propos.

\section{Références}

Benoit, H. (2014a). Éducation des jeunes sourds: interférence et hybridation des discours autour de la figure du déni. La nouvelle revue de l'adaptation et de la scolarisation, 64, 117-133.

Benoit, H. (2014b). Les dispositifs inclusifs: freins ou leviers pour l'évolution des pratiques. La nouvelle revue de l'adaptation et de la scolarisation, 65, 189-204.

Benoit, H. (2014c). Politiques publiques, professionnalités et langages: les maillons faibles de la chaîne inclusive. La nouvelle revue de l'adaptation et de la scolarisation, 67, 181-191.

Benoit, H. (2013). Distorsion et détournement des dispositifs inclusifs: des obstacles à la transition vers de nouvelles pratiques? La nouvelle revue de l'adaptation et de la scolarisation, 61, 49-63.

Benoit, H. (2009). LSF: une discipline scolaire est née, La nouvelle revue de l'adaptation et de la scolarisation, Hors série 5, "L'éducation inclusive en France et dans le monde ", H. Benoit et É. Plaisance (dir.), 175-183.

Benoit, H. (2003). Langue des signes française. Quels enjeux pédagogiques?, La nouvelle revue de I'AIS, 23, 113-122.

Benvenuto, A., Karacostas, A. (2012). Surdité et langue des signes: analyseurs politiques, philosophiques et sociolinguistiques, Base de données des enseignements et séminaires de l'EHESS. http://www.ehess.fr/fr/enseignement/ enseignements/2012/ue/62/

Bon, N. (2004). La logique du fantasme, intervention au séminaire de l'Association lacanienne internationale, Paris, 26-29 août 2004, http://www.freud-lacan.com/ articles/article.php?url_article=nbon100904\#notes.

Compte rendu du congres international pour l'amélioration du sort des sourds-muets, tenu à Milan du 6 au 11 septembre 1880 (1881). Rome, Imprimerie Héritiers Botta. Conseil d'État (2006). Sécurité juridique et complexité du droit, Rapport public. 
Cuxac, C. (2000). La Langue des Signes Française; les Voies de I'lconicité, Faits de Langues. Paris: Ophrys.

Dalle-Nazebi, S., Garcia, B., Kerbourc'h, S. (2009). Vers une redéfinition des territoires: lieux et réseaux de transmission de la Langue des signes française (LSF). In P. Sauzet et F. Pic (dir.), Politique linguistique et enseignement des "langues de France ", Actes du colloque organisé par I'AULF (Association universitaire des langues de France, Inalco), Toulouse, 26-27 mai 2005. L'Harmattan, 223-236. Encrevé, F. (2008). Réflexions sur le congrès de Milan et ses conséquences sur la langue des signes française à la fin du XIXe siècle. Le Mouvement Social, 223(2), 83-98.

Foucault, M. (2001a). Dits et écrits II, 1976-1988, Defert, D. et Ewald, F., (eds). Paris: Quarto Gallimard, texte $n^{\circ} 206,298-329$.

Foucault, M. (2001b). Crise de la médecine ou crise de l'anti médecine, Dits et écrits II, 1976-1988, Defert, D. et Ewald, F., (eds), Paris: Quarto Gallimard, 40-58. Foucault, M. (1966-2008). Les mots et les choses. Gallimard.

Gardou, C. (2005). Fragments sur le handicap et la vulnérabilité. Toulouse: Érès. Gardou, C., Kerlan, A. (2002). L'éthique à l'épreuve du handicap. La nouvelle revue de I'AIS, 19, 7-22.

Haute autorité de santé (2009). Surdité de l'enfant: accompagnement des familles et suivi de l'enfant de 0 à 6 ans, hors accompagnement scolaire. Recommandations de bonnes pratiques. 43 p. http://www.has-sante.fr/portail/upload/docs/application/ pdf/2010-03/surdite_de_lenfant_-_0_a_6_ans_-_recommandations.pdf

Laborit, E., Galant, P., Judé, S. (2010). International visual theater (IVT) : la LSF sur le devant de la scène. La nouvelle revue de l'adaptation et de la scolarisation, 49, 59-69.

Mannoni, O. (1969). Je sais bien, mais quand même..., Clefs pour l'imaginaire ou l'Autre Scène. Paris: Seuil.

MEN. (2016). Les conditions de mise en œuvre des projets linguistiques des jeunes sourds et la qualité de leur parcours, Rapport IGEN-IGAS. http://cache. media.education.gouv.fr/file/2016/29/4/2016-017_parcours_linguistiques_571294.pdf Meynard, A. (2010a). Quand une filière de soin fait taire I'enfance Sourde... La nouvelle revue de l'adaptation et de la scolarisation, 49, 21-35.

Meynard, A. (2010b). Soigner et faire taire: essai sur la médicalisation du Sourd et de sa parole. Toulouse: Érès.

Meynard, A. (2008). Surdité, I'urgence d'un autre regard. Toulouse: Érès.

Meynard, A. (1995). Destins du gestuel. Cliniques méditerranéennes 47-48, 245-262.

Roubier, P. (1955). G. Ripert, Les forces créatrices du droit [note bibliographique]. Revue internationale de droit comparé, 7(4), 877-878.

Séguillon, D. (1994). L'éducation de l'écolier sourd à l'Institution nationale de Paris. Une histoire "à corps et à cri " 1794-1994, Bicentenaire de I'Institut national de jeunes sourds de Paris. Paris: INJS de Paris éditeur, $82 \mathrm{p}$.

Stiker, H.-J. (2003). Corps infimes et sociétés. Paris: Dunod.

Stiker, H.-J. (1999). Quelle lecture du mouvement démocratique peut être fait à partir de la révision de la $\mathrm{ClH}-2$ ? Handicap, 81, 76-84. 
Warsmann, J.-L. (2008). Rapport sur la qualité et la simplification du droit. Commission des lois constitutionnelles, de la législation et de l'administration générale de la République à I'Assemblée Nationale (http://www.cnrs.fr/aquitaine/ IMG/pdf/RapportWarsmann.pdf). 